Most wanted

Science http://doi.org/wdr (2014)

The Majorana fermion - the hypothetical particle that is its own antiparticle - remains as controversial and elusive as its namesake.

The search for Majorana fermions in the form of quasiparticle bound states in condensedmatter systems, fuelled by their possible use as topological qubits, has been underway for some time now. In 2012, the first sightings were reported in a semiconductor nanowire in contact with a superconducting electrode. But the initial excitement was tempered by the possibility that the signal was actually due to the Kondo effect or disorder.

Stevan Nadj-Perge and colleagues took the hunt for Majorana fermions to new territory: a chain of iron atoms on a superconducting lead surface. The ferromagnetic interaction between the atoms, and the strong spin-orbit coupling of the superconductor, provide the necessary ingredients for topological superconductivity. The enhanced resolution of scanning tunnelling microscopy measurements allowed the team to extract strong evidence for the existence of Majorana fermions. But whether the search is now finally over remains to be seen.

\section{Hot and bothered}

Nature Nanotech. http://doi.org/wdq (2014)

Continued device miniaturization means understanding the properties of molecular junctions is becoming ever more important. But thermoelectric effects are difficult to probe in these junctions, as thermal gradients cannot easily be created on such small scales, despite intense research efforts on charge transport. Youngsang Kim and colleagues have now built a device capable of generating large temperature gradients - with an electronic structure that can be actively tuned.
The quintessential set-up for probing effects related to charge is a three-terminal geometry, in which a gate electrode can regulate the current flowing between the source and drain electrodes. Guided by modelling, Kim et al. designed a threeterminal structure, based on a gold nanogap junction, in which sufficient thermal resistance between the source and the drain allows sizeable temperature gradients to form. Using an aluminium gate, the authors revealed the relationship between the electronic structure and the thermoelectric properties for two prototypical molecular junctions.

\section{Long live the proton}

Phys. Rev. D 90, 072005 (2014)

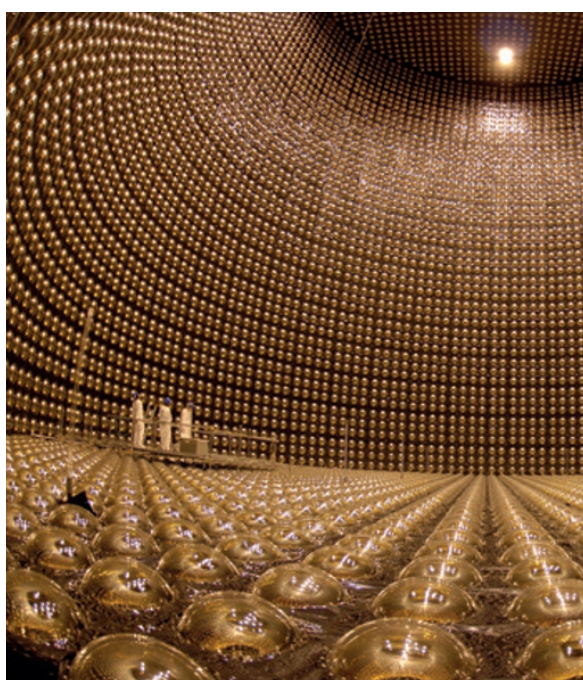

Attempts to unify our understanding of strong and electroweak forces are hampered by the fact that the energies involved are too high to be probed using accelerators. But unified theories also predict nucleon decay

\section{Novel observations}

Nature http://dx.doi.org/10.1038/nature13834 (2014)

Any explosion tends to be most spectacular shortly following detonation, but in the case of a stellar nova, you first have to find it. As luck would have it, amateur nova-hunter Koichi Itagaki spotted Nova Delphini 2013 on 14 August 2013, nine hours after eruption and two days before it reached peak brightness. By day six it was fading. But amateurs and professionals had made plenty of observations by then. In particular, Gail Schaefer and colleagues collected near-infrared data from the day after the explosion until 43 days after, which enabled them to study the expanding fireball in unprecedented detail.

Using the optical/infrared interferometer on Mount Wilson, California, the team tracked the expansion of the ejecta, which can be modelled as a circular core (the 'photosphere' component) surrounded by a circular halo. The two components were equally bright during the first two nights, with the halo growing relatively fainter thereafter, before brightening near the end. These data (days 2-9) show marked asymmetry, suggesting a bipolar shape of the expanding envelope, which may be inherent for novae explosions. where the standard model does not, turning the spotlight - or search light - to proton decay experiments. After nearly two decades on the case, we still have no evidence of nucleon decay, but the Super-Kamiokande Collaboration has now set a new limit on the lifetime of a proton.

The search involved four separate phases, spanning April 1996 to February 2013, carried out in a large water Cherenkov detector (pictured), which is buried beneath Mount Ikenoyama to reduce cosmic background radiation. The past decade of the experiment witnessed a number of improvements, including a reduction in background radiation and a massive increase in efficiency. The results of the first phase of detection, published in 2005, set a preliminary lower limit on proton lifetime. The new figure is more than twofold the original estimate - placing strong constraints on unified theories, and in particular, those incorporating supersymmetry.

\section{Smart photons}

\section{Phys. Rev. X 4, 031056 (2014)}

Random numbers form the basis of certain cryptography protocols. Yet generating a truly random sequence of numbers is not easy. Computer algorithms can produce numbers that are seemingly random, but such pseudo-random numbers are ultimately predictable and hence hackable. Genuine randomness can be found in nature, though: many light sources release photons at random time intervals.

Bruno Sanguinetti and colleagues have now shown that present-day commercial digital cameras and smartphones are sufficiently sensitive to detect streams of photons for conversion into random number sequences. The authors performed experiments with a light-emitting diode coupled to either a type of CCD detector used by astronomers or a smartphone camera. The numbers obtained from the two experiments passed a range of randomness tests - notably, the sequences had vanishing autocorrelations, indicating that they don't exhibit repetitive patterns.

As photon generation in a light-emitting diode is a quantum effect, Sanguinetti et al. call their scheme quantum random number generation. They estimate feasible random bit rates of one megabit per second enough for basic cryptography tools. Expect a quantum random number generator app soon.

Written by May Chiao, Luke Fleet, Iulia Georgescu, Abigail Klopper and Bart Verberck. 